\title{
Differential Labeling of Free and Disulfide-Bound Thiol Functions in Proteins
}

\author{
Bettina Seiwert, ${ }^{\mathrm{a}, *}$ Heiko Hayen, ${ }^{\mathrm{b}}$ and Uwe Karst ${ }^{\mathrm{a}, *}$ \\ ${ }^{a}$ Chemical Analysis Group and MESA ${ }^{+}$Institute for Nanotechnology, University of Twente, Enschede, \\ The Netherlands \\ b ISAS Institute for Analytical Sciences, Dortmund, Germany
}

A method for the simultaneous determination of the number of free cysteine groups and disulfide-bound cysteine groups in proteins has been developed based on the sequential labeling of free and bound thiol functionalities with two ferrocene-based maleimide reagents. Liquid chromatography/electrochemistry/mass spectrometry was used to assign the $\mathrm{N}$-(2ferroceneethyl)maleimide (FEM) labeled free cysteine functionalities in a tryptic digest mixture, whereas a precursor ion scan enables the detection of peptides with ferrocenecarboxylic acid-(2-maleimidoyl)ethylamide (FMEA) labeled disulfide-bound cysteine groups after reduction. Fragment spectra of the labeled peptides yield an excellent coverage of b-type and y-type ions. The ferrocene labeled cysteines were fragmented as $412 \mathrm{Da}$ (FEM) and $455 \mathrm{Da}$ (FMEA). These fragment masses are significantly higher than unlabeled amino acids or dipeptides and are easily detected. The position of free and disulfide-bound cysteine may therefore be assigned in an amino acid sequence. (J Am Soc Mass Spectrom 2008, 19, 1-7) @ 2008 American Society for Mass Spectrometry

$\mathrm{T}$ The presence of cysteine residues in peptides and proteins is the base for building disulfide bridges. This common post-translational modification has a strong influence on the three-dimensional structure of proteins, and it is essential for their function. Free cysteines may participate in substrate binding and catalysis [1]. Therefore, the determination of the number and position of both bound and free cysteine residues may provide important information allowing the understanding of protein structure and function.

Reactions of sulfhydryl groups with other compounds in solution are a common problem in detecting peptides that contain free cysteine residues. A general approach to overcome this problem is to derivatize the thiol groups with iodoacetamide [2], iodoacetic acid [3], or 4-vinylpyridine [4]. However, labeling strategies based on alkylhalides and haloacetamides may exhibit a cross reactivity towards histidine, tyrosine, and methionine [5]. Maleimide-based derivatizing agents are, depending on the $\mathrm{pH}$, more selective for thiol groups. There are two common strategies for the determination of disulfide bonds and free cysteine residues. One is based on the derivatization of the free cysteines and enzymatic cleavage of the protein, followed by the analysis of the resulting fragments containing the intact native disulfide bonds. The second uses the comparison

Address reprint requests to Prof. U. Karst, Institute of Inorganic and Analytical Chemistry, University of Münster, Corrensstr. 30, 48149 Münster, Germany. E-mail: uk@uni-muenster.de

* Current address: University of Münster, Institute of Inorganic and Analytical Chemistry, Corrensstr. 30, 48149 Münster, Germany. E-mail: uk@unimuenster.de of peptide maps of the reduced and the native protein [6]. Suitable enzymes and digestion conditions are critical issues in this case. Another promising approach is to combine derivatization and chemical cleavage at cysteine residues by 2-nitro-5-thiobenzoic acid (NTCB) [7]. However, it is more difficult to obtain structural fragments from the process of collision-induced dissociation (CID) for peptides containing disulfide bonds than for reduced and alkylated ones [8]. Therefore, cysteine alkylation is required to ensure maximum coverage [9]. To overcome these problems, a differential labeling may be employed, using two different derivatizing agents before and after the reduction of the disulfide bonds [10]. Together with LC/MS/MS data of the unreduced protein, bio-informatics enables the calculation of the number and positions of disulfide bonds and free cysteines. In combination with partial reduction by tris-(2-carboxyethyl)phosphine (TCEP), complex disulfide bond patterns can be analyzed [11]. However, complex data analysis has to be employed to determine the complete amino acid sequence. Selective analysis of cysteine residues enables the reduction of the amount of data. For the selective analysis, there are two strategies: selective enrichment or selective detection. The analysis after selective enrichment can be achieved by quaternary amine tags [12], a method based on avidin and biotin [13] or by the covalent capturing of cysteine-containing peptides with pyridyl disulfide reactive groups on agarose beads [14]. Fluorescence may be used as a selective method to detect the derivatized peptides [15]. Although the analysis of cysteine residues and disulfide bonds is simplified, the 
selective analysis of cysteines was, to the best of our knowledge, never applied in combination with the differential labeling approach. We describe a new approach of differential protein labeling using two ferrocene-based derivatizing agents. In a recent paper, we reported the development of a LC/MS method based on the derivatization of thiol groups with the derivatizing agent $\mathrm{N}$-(2-ferroceneethyl)maleimide (FEM) [16]. The method presented in this paper uses a two-reagent differential labeling of thiol and disulfide functions in proteins. The combination of electrochemistry and precursor ion scan mass spectrometry allows the selective analysis of the cysteine containing peptides and simplifies the analysis of complex tryptic digest mixtures without applying dedicated bio-informatic tools.

\section{Experimental}

\section{Chemicals}

All reagents and chemicals were obtained from Sigma Aldrich (Steinheim, Germany) or Fluka Chemie GmbH (Buchs, Switzerland) in the highest quality available and were used without further purification. The water used for HPLC was purified using a Milli-Q Gradient A 10 system and filtered through a $0.22 \mu \mathrm{m}$ Millipak 40 (Millipore, Billerica, MA). The acetonitrile (ACN) for HPLC was obtained from Merck KGaA (Darmstadt, Germany). N-(2-ferrocene-ethyl)maleimide (FEM) was synthesized from ferrocenylmethyltrimethylammonium iodide (obtained from Alfa Aesar, Karlsruhe, Germany) according to a method described in literature $[16,17]$. Ferrocenecarboxylic acid-(2-maleimidoyl)ethylamide (FMEA) was synthesized in a two-step reaction from ferrocenecarboxylic acid (obtained from Alfa Aesar) according to Karst et al. [18, 19]. TPCK treated trypsin from bovine pancreas (12.4 units $/ \mathrm{mg}$ ) from Sigma Aldrich (Steinheim, Germany) was used.

\section{Differential Derivatization}

Stock solutions were prepared of the following compounds: FEM (20 $\mathrm{mM}$ in $\mathrm{ACN})$, ammonium acetate $\left(\mathrm{NH}_{4} \mathrm{OAc}\right)$ buffer $(100 \mathrm{mM}, \mathrm{pH} 4)$, ammonium bicarbonate $\left(\mathrm{NH}_{4} \mathrm{HCO}_{3}\right)$ buffer $(1 \mathrm{M}, \mathrm{pH} 7.8)$, and tris-(2carboxyethyl)phosphine (TCEP) $(100 \mathrm{mM})$ in $0.3 \mathrm{M}$ aqueous ammonia solution. $\alpha$-Lactalbumin, $\beta$-lactoglobulin $\mathrm{B}$, and $\beta$-lactoglobulin A were dissolved in $\mathrm{NH}_{4} \mathrm{OAc}$ to form a $0.3 \mathrm{mM}$ solution. The differential derivatization was carried out by adding $100 \mu \mathrm{L}$ FEM to $1.5 \mathrm{~mL}$ protein solution. Directly after adding the derivatizing agent, $0.72 \mathrm{~g}$ of urea was dissolved and $50 \mu \mathrm{L} \mathrm{NH}_{4} \mathrm{HCO}_{3}$ buffer $(1 \mathrm{M})$ was added to adjust the $\mathrm{pH}$ to 7 . After mixing, $10 \mu \mathrm{L}$ formic acid was added to decrease the $\mathrm{pH}$ value to acidic conditions. The protein solution was passed through a PD-10 desalting column (Amersham Bioscience, Freiburg, Germany) to remove the excess of the reagent. Formic acid $(0.01 \%$ in water) was used as mobile phase. Urea $(0.48 \mathrm{~g})$ was dissolved in $1 \mathrm{~mL}$ protein solution and $50 \mu \mathrm{L} \mathrm{NH}_{4} \mathrm{HCO}_{3}$ solution $(1 \mathrm{M})$ was added. Reduction of the disulfide bonds was performed by adding $50 \mu \mathrm{L}$ TCEP and allowing it to react for $30 \mathrm{~min}$. Afterwards, $200 \mu \mathrm{L}$ FMEA was added and the solution was acidified again and passed through a PD column as described above.

\section{Tryptic Digest}

First, a stock solution of trypsin $(1 \mu \mathrm{g} / \mathrm{mL}$ in $0.01 \%$ formic acid, diluted 1:10 with $100 \mathrm{mM} \mathrm{NH_{4 }} \mathrm{HCO}_{3}$ immediately before use) was prepared. For the insolution digest, $150 \mu \mathrm{L}$ of the trypsin solution was added to $200 \mu \mathrm{L}$ of the desalted protein ( $\beta$-lactoglobulin A) solution containing $\sim 0.03 \mathrm{mM}$ protein in $0.01 \%$ formic acid (trypsin:protein ratio of 1:20 $\{\mathrm{wt} / \mathrm{wt}\})$, and the mixture was incubated overnight at $37^{\circ} \mathrm{C}$.

\section{HPLC/MS/MS}

The LC/MS setup comprised a Shimadzu (Duisburg, Germany) LC system and a QTrap mass spectrometer (Applied Biosystems, Darmstadt, Germany), equipped with an electrospray ionization (ESI) source. The LC system consisted of two LC-10ADVP pumps, a DGC14A degasser, a SIL-HTVP autosampler, a CTO-10 AVP column oven, and a SPD-10 AVVP UV detector. The software used for controlling LC and MS was Analyst 1.4.1 (Applied Biosystems). The analytes were ionized in the ESI interface with an ionspray voltage of $5000 \mathrm{~V}$, using 50 psi nebulizer gas and 70 psi dry gas with a temperature of $500{ }^{\circ} \mathrm{C}$. The proteins and tryptic peptides were detected in the positive ion mode using a Q1 scan $(\mathrm{m} / z 550$ to $\mathrm{m} / \mathrm{z} 1400)$. The collision energy was set to $30 \mathrm{eV}$, entrance potential to $10 \mathrm{~V}$, and exit potential to $15 \mathrm{~V}$ for the precursor ion scan. The collision-activated dissociation (CAD) gas was set to medium.

A binary gradient of $\mathrm{ACN}$ and ammonium formate (10 mM, pH 4) was used for the liquid chromatographic separation. The column employed for the HPLC separation was a Discovery BioWidePore C5 column ( $\mathrm{Su}$ pelco, Taufkirchen, Germany) with a particle size of 5 $\mu \mathrm{m}$, a length of $150 \mathrm{~mm}$, and an i.d. of $2.1 \mathrm{~mm}$. The injection volume was $10 \mu \mathrm{L}$. A flow rate of $0.25 \mathrm{~mL} / \mathrm{min}$ was selected. The following gradients were applied for the separation of the untreated (a) and the differentially labeled (b) whey proteins, and for the tryptic peptides, gradient (c) was used (Table 1).

\section{Fragment Ion Spectra}

All fragment ion spectra of the tryptic peptides were carried out using a Finnigan LTQ linear quadrupole ion trap mass spectrometer (Thermo Fisher Scientific, San Jose, CA) equipped with an off-line nano-ESI source. The instrument was operated in the positive ion mode. The parameters were: source voltage, 1.0 to $1.3 \mathrm{kV}$; capillary voltage, $35 \mathrm{~V}$; capillary temperature, $150^{\circ} \mathrm{C}$; 
Table 1. Gradient profiles used for LC separation

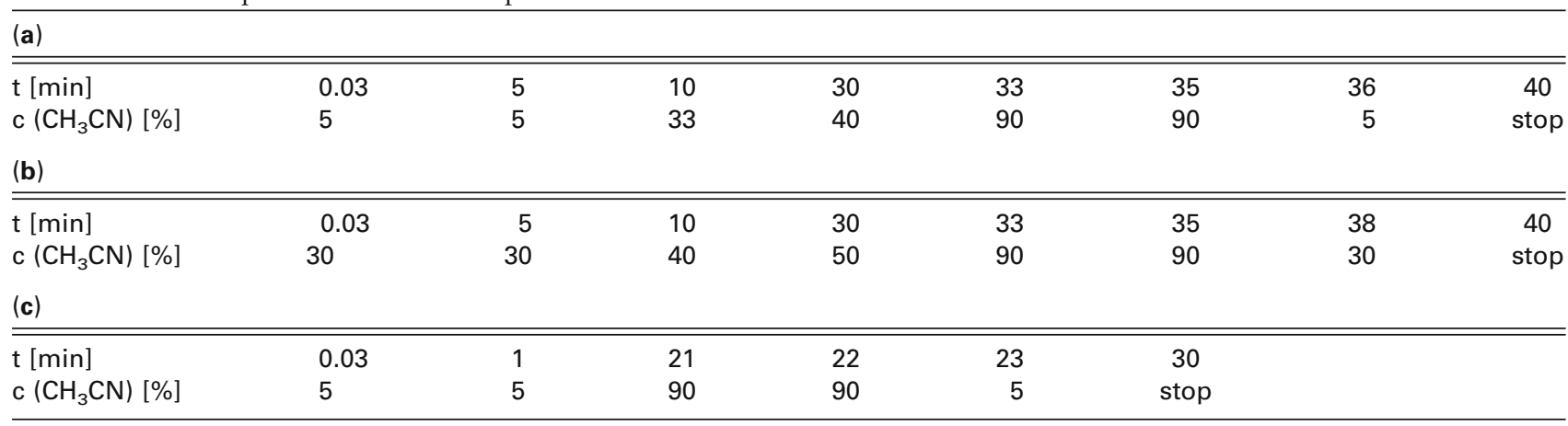

and tube-lens voltage, $110 \mathrm{~V}$. Besides collision-induced dissociation (CID), a new activation technique was used. The pulsed $\mathrm{Q}$ collision-induced dissociation (PQD) eliminates the low mass cut-off of quadrupole ion traps. The normalized collision energies were $22 \%$ for CID and 30\% for PQD, respectively.

\section{LC/EC/MS Analysis}

To hyphenate the LC/MS system with on-line electrochemistry, a conditioning cell model 5021 controlled by a Coulochem II potentiostat from ESA Bioscience Inc. (Chelmsford, MA) was inserted between the outlet of the $\mathrm{UV} / \mathrm{vis}$ detector and the inlet of the ionization interface of the mass spectrometer. To protect the working electrode of the electrochemical cell, a PEEK in-line filter (ESA) was mounted between column and cell.

\section{Cyclic Voltammetry}

FEM and FMEA were dissolved in a mixture of $\mathrm{NH}_{4} \mathrm{HCO}_{3}$ buffer $(100 \mathrm{mM}, \mathrm{pH}$ 7.8) and $\mathrm{ACN}(1: 1$, $\mathrm{vol} / \mathrm{vol}$ ) to form a $0.1 \mathrm{mM}$ solution. After $5 \mathrm{~min}$ of stirring in a nitrogen atmosphere, the stirrer was turned off and cyclic voltammograms were recorded over the potential range from -500 to $1000 \mathrm{mV}$ at a scan rate of $0.35 \mathrm{~V} / \mathrm{s}$. Ferrocene was added to the solution as reference.

\section{Results and Discussion}

\section{Differential Derivatization of Cysteine Residues in Proteins}

In this paper, an approach for differential derivatization with two different derivatizing agents based on ferrocene to characterize free and bound cysteine residues is presented. Two maleimide-based derivatizing agents are used for this purpose. $\mathrm{N}$-(2-ferrocene-ethyl)maleimide (FEM) was already used for the analysis of proteins and peptides by HPLC/MS [16]. The second reagent, ferrocenecarboxylic acid-(2-maleimidoyl)ethylamide (FMEA) was recently introduced [19] for the detection of low molecular weight aminothiols and was synthesized for the differential labeling approach together with FEM.
As previously demonstrated, the ferrocene-based derivatizing agents FEM and FMEA react selectively with sulfhydryl groups of cysteine residues in proteins at a $\mathrm{pH}$ around 7 [19]. The first step of the differential derivatization is the labeling of the free thiol functionalities. This is achieved by simultaneous protein denaturation and derivatization by FEM. The reaction time needed for derivatization is less than $5 \mathrm{~min}$ under the conditions stated above. The excess of FEM has to be removed before the second step (the reaction of the disulfide bound cysteines with FMEA). The difference in size between protein and low molecular weight reagents was utilized for a respective separation on a PD 10 column. Afterwards, TCEP is added to reduce the disulfide bonds in the presence of urea, which denatures the protein to yield accessible thiol functionalities. TCEP is known as an easily soluble and very stable compound, which reduces disulfide bonds more efficiently than DTT. It does not, according to literature, react with other functional groups in proteins [20].

The second step of the differential derivatization is the labeling of the cysteine group of the former disulfide bond. Therefore, FMEA is added as a second derivatizing agent. The sequence of the reagents is selected due to their water solubility. FEM is poorly water-soluble whereas FMEA, due to the additional carboxyl group, is highly soluble in water and may be added in large excess even under aqueous conditions. The excess is needed because TCEP reacts with maleimide as well. However, there is no reduction agent available that does not react with the derivatizing agent. Subsequently, the number of cysteine residues involved in disulfide bonds and the number of the free cysteines can be determined quasi-simultaneously by MS. Therefore, the mass of the untreated and the differentially labeled protein has to be determined by mass spectrometry. The mass tags (352 Da, FMEA and $309 \mathrm{Da}, \mathrm{FEM}$ ) increase the mass of the protein significantly. Furthermore, the mass tag difference (43 Da) is large enough to distinguish between the derivatizing agents even after a reaction with larger proteins.

A mixture of three whey proteins was selected as model compounds to demonstrate the applicability of the method to protein analysis. $\beta$-Lactoglobulin $\mathrm{B}$ and 

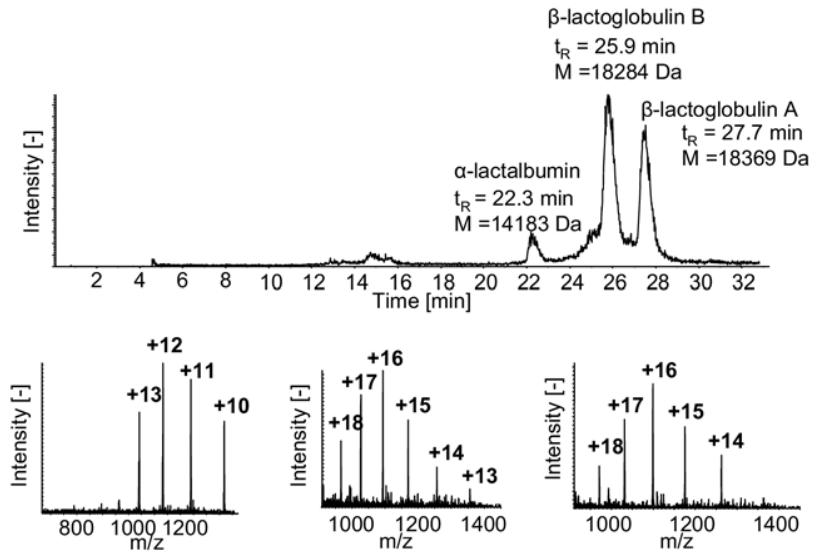

Figure 1. Total ion current (TIC) chromatogram of the separation of the three whey proteins $\alpha$-lactalbumin, $\beta$-lactoglobulin B, and $\beta$-lactoglobulin A. Below: mass spectra of the protein peaks and annotated charge states.

A both contain two disulfide bridges (AA66-AA160 and AA106-AA119) and one free cysteine (AA121) [21]. $\alpha$-Lactalbumin contains four disulfide bridges (AA6AA120, AA28-AA111, AA61-AA77, and AA73-AA91) [22]. The untreated proteins elute from a BiowidePore C5 column (Supelco, Taufkirchen, Germany) in the order $\alpha$-lactalbumin, $\beta$-lactoglobulin B and $\beta$-lactoglobulin A (Figure 1). $\alpha$-Lactalbumin $(14,183 \mathrm{Da})$ is well separated from the other peaks.

$\beta$-Lactoglobulin B $(18,284 \mathrm{Da})$ and A (18,369 Da), which differ in two amino acid (AA) residues (var. A/var. B, AA 64: Asp/Gly and AA 118: Val/Ala) are clearly, although not baseline separated.

After derivatization of $\beta$-lactoglobulin B (18,593 Da) and $\mathrm{A}(18,679 \mathrm{Da})$, a mass gain of $309 \mathrm{Da}$ and $310 \mathrm{Da}$, respectively, was observed. Considering the mass accuracy of the instrument used, one FEM label can unambiguously be assigned to any of the proteins. The mass of $\alpha$-lactalbumin remains unchanged. This is consistent with one free cysteine in the lactoglobulins and no free cysteine in lactalbumin. After the second labeling step, the proteins elute in reversed order: first $\beta$-lactoglobulin $\mathrm{A}$, then $\beta$-lactoglobulin B, and finally $\alpha$-lactalbumin (Figure 2). Deconvolution of the mass spectrum of $\alpha$-lactalbumin provides a mass of $17,009 \mathrm{Da}$. The mass difference of 2826 Da shows that the protein is derivatized by 8 FMEA (2824 Da). Consequently, all labeled cysteines were parts of previous disulfide bonds. The mass spectrum of $\beta$-lactoglobulin B provides a mass of $20,006 \mathrm{Da}$ and the spectrum of $\beta$-lactoglobulin A provides a mass of 20093 Da. The mass difference compared with the unlabeled proteins is $1722 \mathrm{Da}$. This correlates well with four FMEA and one FEM. Therefore, both $\beta$-lactoglobulin A and B contain two disulfide bonds and one free cysteine. As shown for the mixture of whey proteins, it is possible to determine the number of free and bound cysteines of a protein by a single mass spectrometric measurement after the differential derivatization.

\section{Identification of Ferrocene-Labeled Peptides by MS/MS}

To obtain more information about the location of the free cysteines and the disulfide bonds in the protein, a tryptic digest is performed. For the following peptide separation, the ferrocene units have the attractive feature of reducing the polarity of the peptide significantly, thus leading to the separation of the ferrocenecontaining peptides at comparably high organic content (long retention times). Furthermore, a predetermined fragmentation site, an electrochemical active group, and a metal ion is introduced. Thus, three different analytical methods may be applied to determine the ferrocene-derivatized peptides: (1) a precursor ion scan in LC/MS, (2) the combination of LC with electrochemical conversion and MS, and (3) atomic spectroscopy. The latter option is not used within this work, while the other two options are applied as indicated below. The identification of the ferrocene-labeled tryptic peptides is shown for the differentially labeled $\beta$-lactoglobulin A.

The fragment ion spectra of the FMEA derivatives show three common fragment ions at $m / z 129, m / z 185$, and $m / z 213$. The fragment at $m / z 213$ corresponds to the cleavage within the ester function next to the carbonyl carbon atom. The FMEA-containing peptides may therefore be detected by a precursor ion scan with the fragment ion mass of $m / z 213$ (Figure 3).

Beside the TCEP derivative, there are four additional peaks present in the precursor ion scan spectrum. These peptides are labeled by at least one FMEA. The four peptides, a, b, c, and $d$, selected by the precursor ion scan are analyzed in more detail. Amino acid sequence analysis ( $N$-terminal b-type ions and $C$-terminal y-type ions) of the tryptic fragments is possible by obtaining fragment spectra. The ferrocene labels attached to the cysteines were fragmented to yield b-type and y-type ions with a mass of $412 \mathrm{Da}$ (FEM) and $455 \mathrm{Da}$ (FMEA). These fragment masses are significantly higher than unlabeled amino acids or dipeptides and can be easily
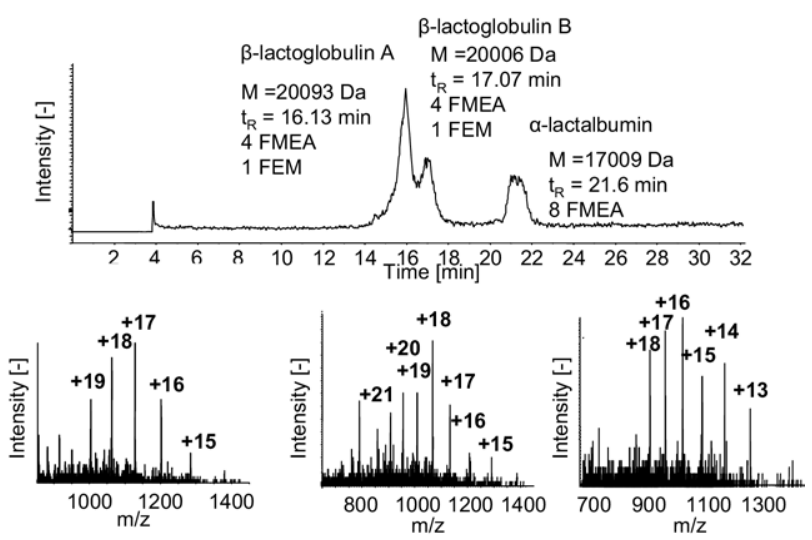

Figure 2. TIC chromatogram of the separation of the differentially labeled proteins $\beta$-lactoglobulin A, $\beta$-lactoglobulin B, and $\alpha$-lactalbumin. Below: mass spectra of the protein peaks and annotated charge states. 


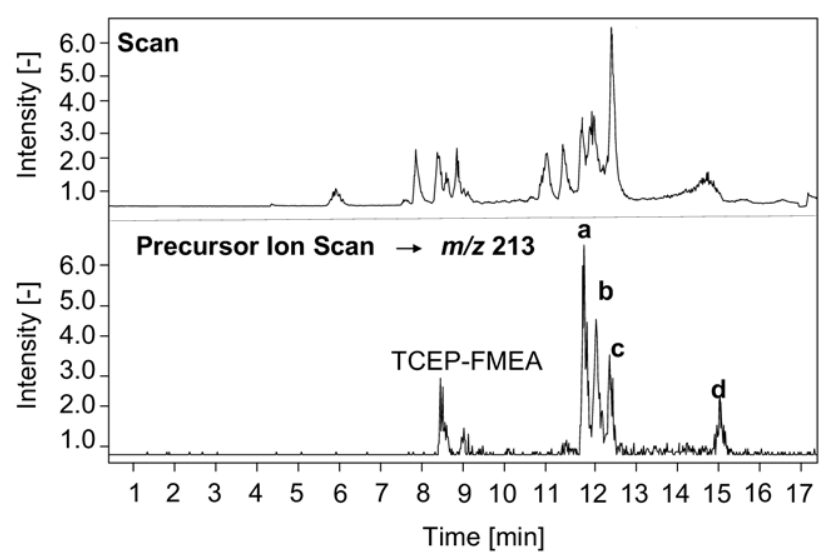

Figure 3. TIC chromatogram of a tryptic digest of $\beta$ lactoglobulin A (upper chromatogram) and the corresponding precursor ion scan (below).

detected. Furthermore, especially singly labeled peptides and fragments exhibit a typical isotopic pattern resulting from the iron atom. The nature and the sequence of the amino acid residues is obtained by comparing the fragment masses obtained by theoretical cleavage of the protein sequence with the measured one. At $t_{R}=11.71 \mathrm{~min}$ a peptide with the mass of 2010 Da elutes with the charge state +2 and +3 (Figure 4a). The tandem mass spectrum of the precursor ion (AA149-162) with $\mathrm{m} / \mathrm{z} 1006.42$ (doubly charged) is shown in Figure 5 . The mass difference $(455.12 \mathrm{Da})$ between fragment $\mathrm{y}_{2}(\mathrm{~m} / \mathrm{z} 269.08)$ and $\mathrm{y}_{3}(\mathrm{~m} / \mathrm{z} 724.20)$ is equal to the mass of one cysteine derivatized by FMEA. Consequently, the cysteine C160 is part of a disulfide bond.

The peptide with a mass of 3898 Da elutes at $t_{R}=$ 12.34 min (Figure 4b, AA41-69). Deconvolution of the peak with $t_{R}=12.45$ min leads to a mass of $3769 \mathrm{Da}$ present in the charge states +3 to +5 (Figure 4c, AA41-70). Due to the mass difference of 129 Da (Lys

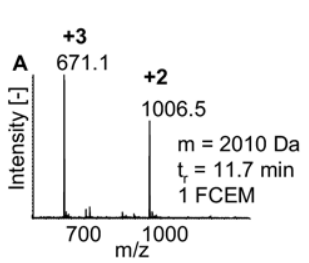

LSFNPTQLEEQCHI

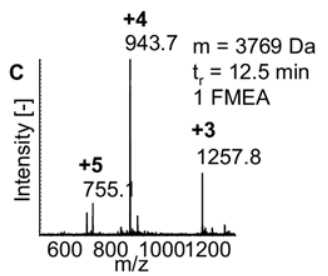

VYVEELKPTPEGDLEILLQKWENDECAQK

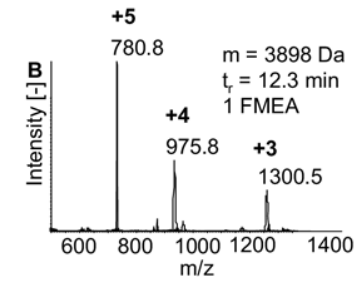

VYVEELKPTPEGDLEILLQKWENDECAQKK

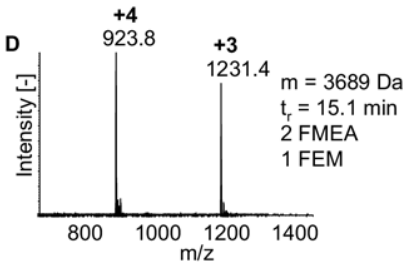

YLLFCMENSAEPEQSLVCQCLVR
Figure 4. Mass spectra of selected tryptic peptides of $\beta$ lactoglobulin $\mathrm{A}$ as identified by precursor ion scan measurements.

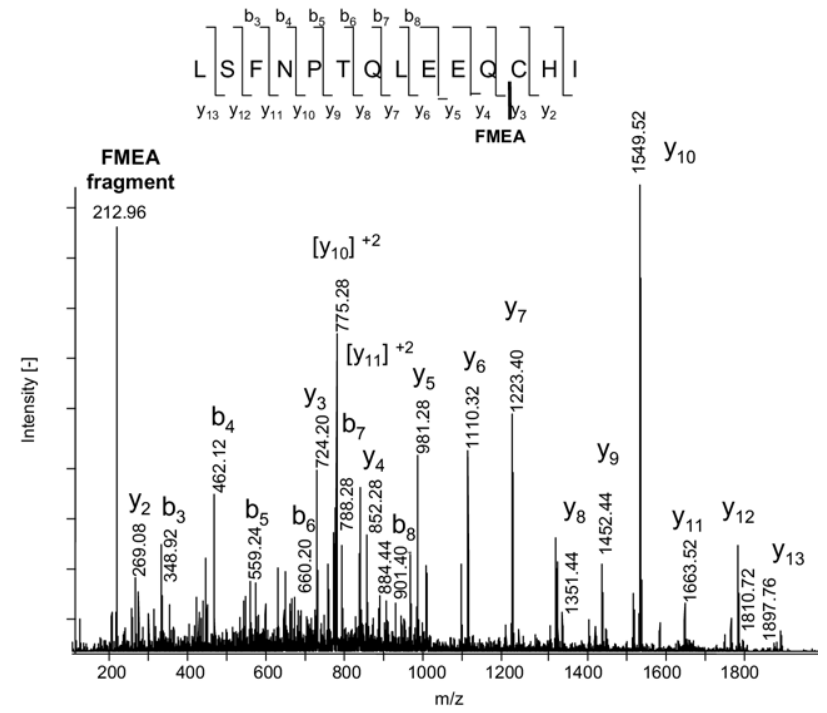

Figure 5. Tandem MS spectrum of the peptide with $\mathrm{m} / \mathrm{z} 1006$ (peptide AA 149-162).

$129 \mathrm{Da})$, peak B corresponds to the miscleaved peptide of peak c. The mass difference between fragment $\mathrm{y}_{4}(\mathrm{~m} / \mathrm{z}$ 801.32) and the triply charged $\mathrm{m} / \mathrm{z} 1257.59$ of peptide $c$ (Figure 6) is equal to the mass of FMEA-labeled Cys (455.12 Da) connected to Ala (71.03 Da), Gln (128.06 Da), and Lys (128.09 Da). Therefore, Cys 66 is a FMEAlabeled cysteine, which indicates that it is bound in a disulfide bond.

Peak D elutes at $t_{R}=15.07 \min (A A 102-124)$. MS/MS analysis of the triply charged tryptic peptide with $m / z 1230.46$ is shown in Figure 7. From the mass loss of 65.1 Da yielding $\mathrm{m} / \mathrm{z} 1208.44$ (triply charged) it is obvious that at least one FEM label is present. A loss of $65 \mathrm{Da}$ is the most abundant fragment of FEM derivatives in tandem MS spectra. It corresponds to the symmetrically coordinated ligand cyclopentadienyl (leaving as a neutral radical). However, neutral loss

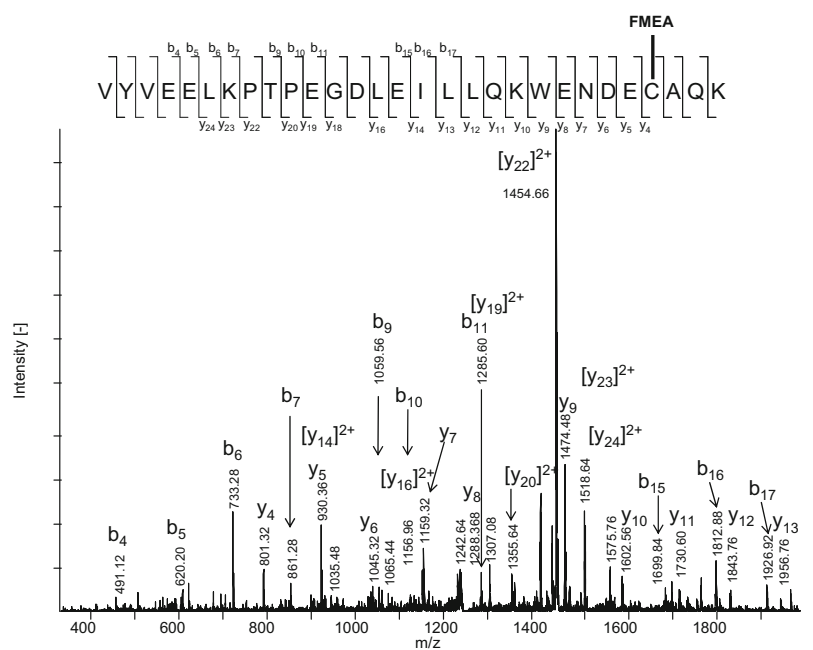

Figure 6. Tandem MS spectrum of the peptide with $\mathrm{m} / \mathrm{z} 1257$ (peptide AA 101-124). 


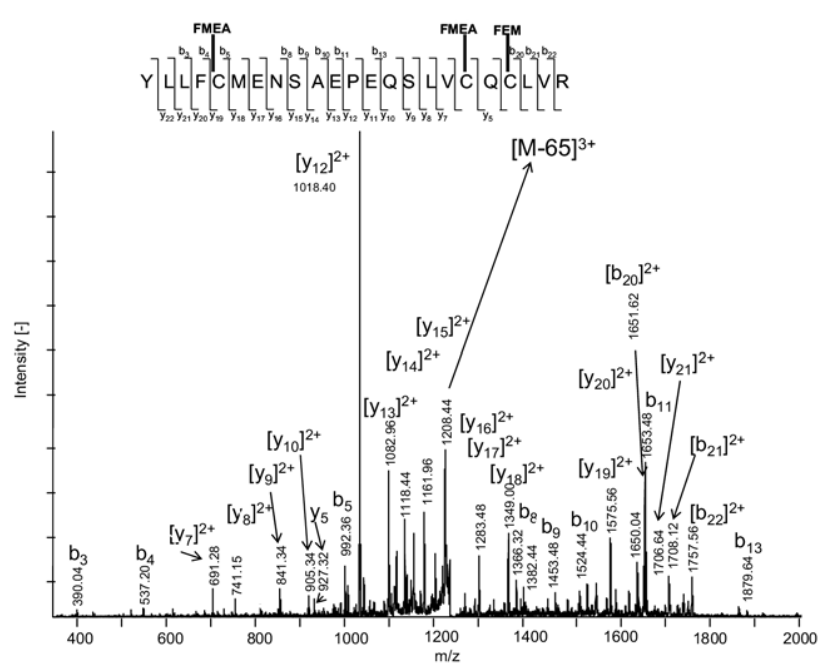

Figure 7. Tandem MS spectrum of the peptide with $\mathrm{m} / \mathrm{z} 1230$ (peptide AA102-124).

scans are not sensitive enough to get additional information. The mass difference of 445.16 Da between fragment $b_{4}(m / z 537.20)$ and $b_{5}(m / z$ 992.36) is consistent with the mass of a cysteine labeled with one FMEA. This demonstrates that Cys106 is a FMEA labeled Cys. Consequently Cys106 is bound in a disulfide bond.

The difference between the fragments $y_{7}(\mathrm{~m} / z$ 691.28, doubly charged) and $\mathrm{y}_{5}(\mathrm{~m} / \mathrm{z}$ 927.32) can be assigned to Cys119, derivatized by FMEA due to the mass difference of 455.12 Da together with an additional Val (99.07 Da). Fragment $b_{20}(m / z 1651.62$, doubly charged) has a mass difference of $387.36 \mathrm{Da}$ compared with the peptide mass (3691.38 Da). The difference of fragment $b_{20}$ and fragment $y_{5}(\mathrm{~m} / \mathrm{z}$ 927.32) is $539.9 \mathrm{Da}$. Referring to Gln $(128.06 \mathrm{Da})$ bound to one FEM-labeled Cys residue (411.81 Da). FMEA labeling is not possible because there is no amino acid with a mass 85.01 Da. Consequently, Cys121 is labeled by FEM, which is consistent with the literature [21]. The free cysteine in the peptide

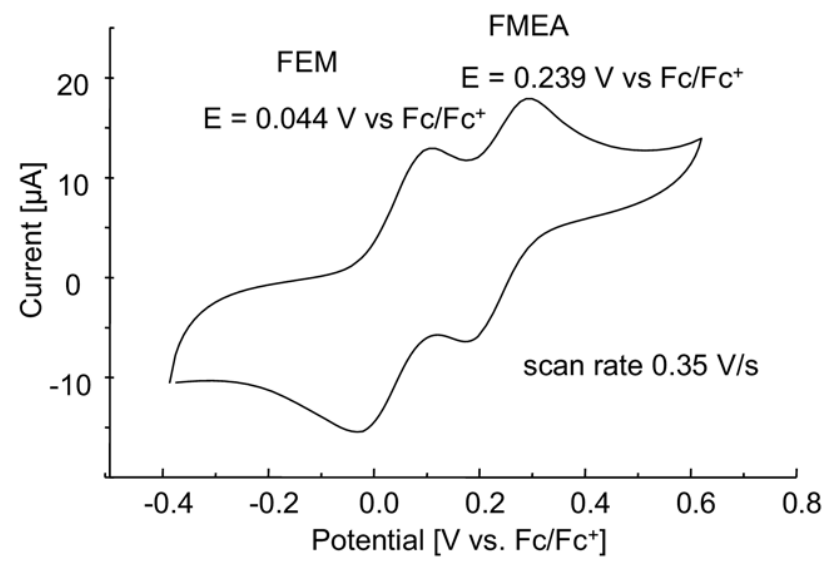

Figure 8. Cyclic voltammogram of a $0.1 \mathrm{mM}$ solution of FMEA and FEM in $100 \mathrm{mM}$ ammonium formate $\mathrm{pH}$ 7.8/acetonitrile (1:1, $\mathrm{vol} / \mathrm{vol})$.

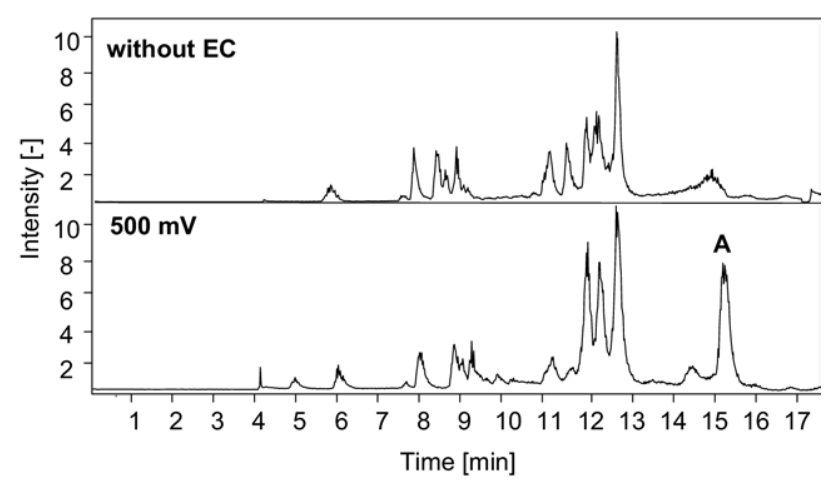

Figure 9. TIC chromatogram of a tryptic digest of $\beta$-lactoglobulin A without and with electrochemical cell $\left(500 \mathrm{mV}\right.$ versus $\left.\mathrm{Pd} / \mathrm{H}_{2}\right)$.

is Cys121. Therefore, it was assigned correctly by our approach.

\section{Identification of Ferrocene-Labeled Peptides by LC/EC/MS}

Figure 8 shows a cyclic voltammogram for a mixture of FMEA and FEM. Compared with FEM, the oxidation potential of FMEA is increased by $200 \mathrm{mV}$. If the carbonyl group is located next to the ferrocene, its electron-withdrawing effect explains the increase. The two methylene groups of FEM directly bound to one cyclopentadienyl $(\mathrm{Cp})$ ring facilitate the oxidation of the ferrocene group by stabilizing the ferrocenium cation with their +I-effect.

For FEM-labeled peptides, it was already shown [17] that it is possible to enhance the intensity of the peak by an on-line electrochemical oxidation. A comparison of LC/MS and LC/EC/MS measurements of a tryptic digest of $\beta$-lactoglobulin $\mathrm{A}$ is shown in Figure 9. The intensity of peptide $\mathrm{A}$ is enhanced significantly. As already known from the MS/MS experiments (see above), peak A corresponds to the tryptic peptide that contains both derivatizing agents (peptide d, Figure 4).

In Figure 10, the behaviors of the tryptic peptide AA102-124, differentially labeled by one FEM and two FMEA (a) is compared with the behavior of the same peptide exclusively labeled by FMEA (b). In both cases, the charge distribution is shifted to higher values com-
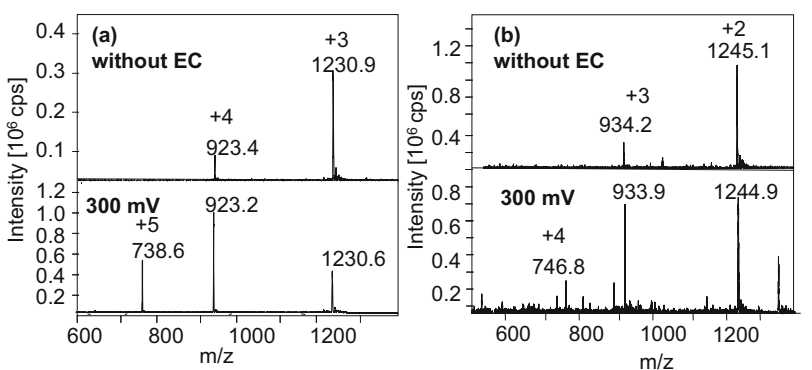

Figure 10. Comparison of the influence of the electrochemical conversion on a peptide labeled by FMEA and FEM (a) to the same peptide that was exclusively labeled by FMEA (b). 
pared with the unlabeled peptide due to electrochemical oxidation/ionization at the ferrocene functions. This behavior is not observed for unlabeled peptides and can be applied to distinguish labeled and unlabeled peptides. As observed in [18], labeling with at least one FEM (a) results in an increase in signal intensity when applying an electrochemical potential. If the same peptide is exclusively labeled by FMEA (b) the signal intensity stays constant or even decreases after electrochemical treatment. This effect is most likely caused by the distribution of the signal intensity over more peaks, which is caused by the electrochemical oxidation.

The LC/EC/MS measurements are useful for the differential labeling, because peptides labeled by both ferrocene reagents are detected by the precursor scan (peptide D, Figure 3) and by the LC/EC/MS approaches (peptide A, Figure 9). Peptides labeled by only one of the derivatizing agents were detected by only one of the methods. Furthermore, the change in charge state distribution does not occur for unlabeled peptides. The identification of peptides by single quadrupole instruments is simplified by a broader charge state distribution. With these instruments, limited information about the charge state is available from the isotopic pattern. In addition, the peaks are shifted towards smaller $m / z$ values, which is advantageous in case of mass spectrometers with a limited mass range.

\section{Conclusion}

A differential derivatization procedure for free and disulfide-bound thiol functionalities in proteins based on two maleimide reagents with ferrocene groups has been introduced. Tandem mass spectrometry and EC/MS may be used to assign the individual cysteine residues as free or thiol-bound. The method may be used for intact proteins or their tryptic digests.

In the future, the differential labeling strategy may be combined with partial reduction by TCEP, which will be useful for the analysis of complex disulfide bridges. TCEP may be used to reduce a particular disulfide bond selectively [11]. The sample amount required for the differential derivatization may be further reduced based on variations in the sample preparation procedure, and the use of capillary LC columns and a nanospray interface would most likely lead to significantly improved limits of detection.

\section{Acknowledgments}

The authors gratefully acknowledge financial support by the Deutsche Forschungsgemeinschaft, Bonn, Germany, and the Fonds der Chemischen Industrie, Frankfurt (Main), Germany.

\section{References}

1. Thornton, J. M. Disulphide Bridges in Globular Proteins. J. Mol. Biol. 1981, 151, 261-287.

2. Gellerfors, E.; Lunden, M.; Nelson, B. D. Evidence for a Function of Core Protein in Complex III from Beef-Heart Mitochondria. Eur. J. Biochem. 1976, 67, 463-468.

3. Rosner, L. The Reaction Between Iodoacetic Acid and Denatured Egg Albumin. J. Biol. Chem. 1940, 132, 657-662.

4. Sebastiano, R.; Citterio, A.; Lapadula, M.; Righetti, P. G. A New Deuterated Alkylating Agent for Quantitative Proteomics. Rapid Commun. Mass Spectrom. 2003, 17, 2380-2386.

5. Wang, W.; Rusin, O.; Xu, X.; Kim, K. K.; Escobedo, J. O.; Fakayode, S. O.; Fletcher, K. A.; Lowry, M.; Schowalter, C. M.; Lawrence, C. M.; Fronczek, F. R.; Warner, I. M.; Strongin, R. M. Detection of Homocysteine and Cysteine. J. Am. Chem. Soc. 2005, 127, 15949-15958.

6. Mhatre, R.; Woodard, J.; Zeng, C. Strategies for Locating Disulfide Bonds in a Monoclonal Antibody via Mass Spectrometry. Rapid Commun. Mass Spectrom. 1999, 13, 2503-2510.

7. Codina, A.; Vilaseca, M.; Tarrago, T.; Fernandez, I.; Ludevid, D.; Giralt E. Location of Disulfide Bonds in Mature $\alpha$-L-Fucosidase from Pea. J. Pep. Sci. 2001, 7, 305-315.

8. Gorman, J. J.; Ferguson, B. L.; Speelman, D.; Mills, J. Determination of the Disulfide Bond Arrangement of Human Respiratory Syncytial Virus Attachment (G) Protein by Matrix-Assisted Laser Desorption/ Ionization Time-of-Flight Mass Spectrometry. J. Protein Sci. 1997, 6, $1308-1315$

9. Sechi, S.; Chait, B. T. Modification of Cysteine Residues by Alkylation. A Tool in Peptide Mapping and Protein Identification. Anal. Chem. 1998, 70, 5150-5158.

10. Yen, T.-Y.; Joshi, R. K.; Yan, H.; Seto, N. O. L.; Palcic, M. M.; Macher, B. A. Characterization of Cysteine Residues and Disulfide Bonds in Proteins by Liquid Chromatography/Electrospray Ionization Tandem Mass Spectrometry. J. Mass Spectrom. 2000, 35, 990-1002.

11. Yen, T.-Y.; Yan, H.; Macher, B. A. Characterization of Closely Spaced, Complex Disulfide Bond Patterns in Peptides and Proteins by Liquid Chromatography/Electrospray Ionization Tandem Mass Spectrometry. J. Mass Spectrom. 2002, 37, 15-30.

12. Ren, D.; Julka, S.; Inerowicz, H. D.; Regnier, F. E. Enrichment of Cysteine-Containing Peptides from Tryptic Digests Using a Quarternary Amine Tag. Anal. Chem. 2004, 76, 4522-4530.

13. Gygi, S. P.; Rist, B.; Gerber, S. A.; Turecek, F.; Gelb, M. H.; Aebersold, R. Quantitative Analysis of Complex Protein Mixtures Using IsotopeCoded Affinity Tags. Nat. Biotechnol. 1999, 17, 994-999.

14. Shen, M.; Guo, L.; Wallace, A.; Fitzner, J.; Eisenman, J.; Jacobsen, E.; Johnson, R. S. Isolation and Isotope Labeling of Cysteine- and Methionine-Containing Tryptic Peptides. Mol. Cell. Proteomics 2003, 315-324.

15. Masuda, M.; Toriumi, C.; Santa, T.; Imai, K. Fluorogenic Derivatization Reagents Suitable for Isolation and Identification of Cysteine-Containing Proteins Utilizing High-Performance Liquid Chromatography-Tandem Mass Spectrometry. Anal. Chem. 2004, 76, $728-735$.

16. Seiwert, B.; Karst, U. Analysis of Cysteine-Containing Proteins Using Precolumn Derivatization with N-C2-Ferroceneethyl)-Maleimide and LC/Electrochemistry/MS. Anal. Bioanal. Chem. 2007, 388, 1633-1642.

17. Shimada, K.; Oe, T.; Nambara, T. Sensitive Ferrocene Reagents for Derivatization of Thiol Compounds in High-Performance Liquid Chromatography with Dual-Electrode Coulometric Detection. J. Chromatogr. Biomed. Appl. 1987, 419, 17-25.

18. Diehl, G.; Liesener, A.; Karst, U. Liquid Chromatography with Postcolumn Electrochemical Treatment and Mass Spectrometric Detection of Nonpolar Compounds. Analyst 2001, 126, 288-290.

19. Seiwert, B.; Karst, U. Simultaneous LC/MS/MS Determination of Thiols and Disulfides in Urine Samples Based on Differential Labeling with Ferrocene-Based Maleimides. Anal. Chem. 2007, 79, 7131-7138.

20. Kirey, T. L. Reduction and Fluorescent Labeling of Cyst(e)ineContaining Proteins for Subsequent Structural Analyses. Anal. Biochem. 1989, 180, 231-236.

21. Surroca, Y.; Haverkamp, J.; Heck, A. J. R. Towards the Understanding of Molecular Mechanisms in the Early Stages of Heat-Induced Aggregation of $\beta$-Lactoglobulin AB. J. Chromatogr. A 2002, 970, 275-285.

22. McGuffy, M. K.; Epting, K. L.; Kelly, R. E.; Foegeding, E. A. Denaturation and Aggregation of Three $\alpha$-Lactalbumin Preparation at Neutral pH. J. Agric. Food Chem. 2005, 53, 3182-3190. 